\title{
Les journaux de Waleria Tarnowska et Eliza Michałowska
}

\section{Michel Braud}

\section{(2) OpenEdition}

Journals

\section{Édition électronique}

URL : http://journals.openedition.org/genesis/529

DOI : $10.4000 /$ genesis. 529

ISSN : 2268-1590

Éditeur :

Presses universitaires de Paris Sorbonne (PUPS), Société internationale de génétique artistique littéraire et scientifique (SIGALES)

Édition imprimée

Date de publication : 1 janvier 2011

Pagination : 177-180

ISBN : 978-2-84050-749-9

ISSN : 1167-5101

\section{Référence électronique}

Michel Braud, «Les journaux de Waleria Tarnowska et Eliza Michałowska », Genesis [En ligne],

32 | 2011, mis en ligne le 17 septembre 2012, consulté le 02 mai 2019. URL : http://

journals.openedition.org/genesis/529 ; DOI : 10.4000/genesis.529 


\title{
Les journaux de Waleria Tarnowska et Eliza Michałowska
}

\author{
Michel Braud*
}

I existe, dans des archives publiques polonaises, comme sans doute dans celles d'autres pays de l'est de l'Europe, d'assez nombreux journaux rédigés en français au XVIII e et au XIX ${ }^{\mathrm{e}}$ siècle, tenus par des aristocrates pour lesquels notre langue était la langue de communication usuelle et la langue de la culture. Ils restent encore mal connus et n'ont pas fait à ma connaissance l'objet d'un dépouillement systématique. Il s'agit pour l'essentiel de journaux de voyage, en Italie et en France particulièrement, qui s'inscrivent dans le cadre de l'intérêt pour les lieux, les techniques et les sociétés, qui se développe à cette époque 1 .

Quelques journaux de jeunes femmes se détachent toutefois de cet ensemble. J'en évoquerai deux qui se trouvent à la bibliothèque de l'Université Jagellonne de Cracovie, et qui présentent des caractères à la fois typiques et complémentaires ${ }^{2}$.

Le premier est un journalchronique tenu par la comtesse Waleria ze Stroynowskich Tarnowska ${ }^{3}$, de 1803 à 1838 soit, pour elle, de vingt et un à cinquante-six ans.

Née en 1782, la comtesse Tarnowska appartient par son père, Walerian Stroynowski, à cette aristocratie polonaise qui possède d'importantes propriétés en Russie, et elle a épousé, en 1799, Jan Feliks Tarnowski, héritier d'une des grandes familles de la noblesse polonaise. Elle partage d'ailleurs son temps entre le château de son mari, près de Tarnobrzeg, en Galicie sous domination autrichienne ${ }^{4}$, et sa propriété en Volhynie, dans l'empire russe (actuelle Ukraine) ${ }^{5}$.

Elle commence à tenir son journal au début de son voyage de noces en Italie, en octobre 1803, et le dédie à sa fillette de neuf mois qu'elle laisse aux soins d'une nourrice :

Tarnow, 5 octobre 1803. - Ma Rosalie, mon enfant, ma chère enfant, je t'ai quittée! tu ne sens pas encore mon

*. Michel Braud est professeur de littérature française à l'université de Pau et des Pays de l'Adour. Ses travaux portent sur l'autobiographie et le journal personnel. Il est l'auteur de La Forme des jours. Pour une poétique du journal personnel (Paris, Éditions du Seuil, coll. « Poétique », 2006) et de La Tentation du suicide dans les écrits autobiographiques (Paris, PUF, 1992). Il a codirigé plusieurs volumes collectifs : Où en sommes-nous dans les recherches autobiographiques (Cracovie, WSP, 1993) ; Jacques Borel, l' imagination autobiographique (Paris, Minard, 2000) ; Écritures du ressassement (Bordeaux, Presses universitaires de Bordeaux, 2001) ; L'Irressemblance. Poésie et autobiographie (Bordeaux, Presses universitaires de Bordeaux, 2007) ; Poétiques de la durée (Bordeaux, Presses universitaires de Bordeaux, 2010). absence... ah ! combien je sens la tienne! Mais un jour ton jeune cœur m'entendra, me répondra... Je vais donc, en voyageant, écrire ici, pour toi, tout ce qui m'intéressera davantage.

Au long de son voyage, elle s'adresse de temps à autre à son enfant, même si elle tient visiblement son journal pour conserver la trace de

1. Par exemple, à la bibliothèque de l'Université Jagellonne de Cracovie, les Journeaux des voyages par m. le comte Michel Mniszek 1767$1768: 1$. Observations sur Rome et l'Italie contenues dans ce vollume faites depuis le 15 bre au $1510 b r e 1767$; 2 . Observations contenues dans ce vollume faites depuis le 20 10bre 1767 jusqu'au 3 juin 1768 ; 3. Routes contenues dans ce vollume. De Rome, Varsovie, à Wisnowiec. Depuis le 15 10bre 1767 jusqu' au juillet 1768.

2. Je remercie Claire Devèze de m'avoir signalé l'existence de ces deux manuscrits.

3. Mes Voyages, deux volumes manuscrits, bibliothèque de l'Université Jagellonne de Cracovie, cotes 121/52 et 122/52 ; Mon Journal, neuf volumes manuscrits, cotes 112/52 à 120/52. 4. Rappelons qu'au début du XIXe siècle, la Pologne se trouve partagée entre la Prusse (à l'ouest), l'Autriche (au sud) et la Russie (à l'est), et que la partie centrale, appelée Royaume du Congrès, ne conserve que peu de temps un semblant d'autonomie sous la férule russe.

5. Elle est l'un des cent plus importants propriétaires terriens des trois provinces de Volhynie, de Podolie et de Kiev. Voir Daniel Beauvois, Le Noble, le serf, et le révizor : la noblesse polonaise entre le tsarisme et les masses ukrainiennes (1831-1863), Montreux, Éditions des archives contemporaines, 1985 , p. $308-309$ 
son périple. La comtesse décrit en détail les monuments qu'elle visite, les tableaux et sculptures qu'elle a l'occasion d'admirer, et plus largement les villes et paysages qu'elle traverse. Elle rend compte des contacts qu'elle établit avec des savants (Felice Fontana) ou des artistes (Antonio Canova), mais n'est pas indifférente non plus à l'actualité politique, notamment lorsque celleci concerne la Pologne partagée. Lorsqu'elle apprend, le 17 juin 1804, la mort de son enfant, survenue le 4 avril, les notes se raccourcissent et s'espacent, et les évocations sont pour l'essentiel en relation directe avec l'état d'esprit de la jeune femme.

Ce journal de voyage est rédigé sur deux cahiers oblongs à l'italienne d'environ 10,5 sur $17 \mathrm{~cm}$, de soixante-trois et soixante et onze folios. Le texte couvre le volume I et les cinquante-neuf premiers folios du volume II. Il est visiblement écrit au fil de la plume et comporte peu de ratures sinon, après coup, sur les passages rédigés après qu'elle a reçu l'annonce de la mort de sa fille. Quelques corrections, d'une encre différente, signalent une relecture attentive. Waleria Tarnowska a aussi ajouté des notes après le texte (sur les folios 60 à 71 du second cahier), numérotées NB1 à NB90, et qui sont appelées dans le texte par cette référence. Ces notes, rédigées après son retour en Pologne, complètent certaines informations, corrigent ou nuancent les jugements portés dans le journal. Quelques gravures de monuments ont été insérées hors texte, et reliées avec le journal.
Ce manuscrit du journal de voyage en Italie a fait l'objet d'une publication en deux temps. Le début a paru en 1924-1925 dans plusieurs numéros successifs de la Revue de Pologne, avec une préface de Georges Mycielski, arrière-petit-fils de la comtesse, qui raconte notamment comment il a sauvé ces carnets, comme l'un de ses biens les plus précieux, devant l'avancée du front russe en 1914. La fin a été éditée dans le bulletin de la bibliothèque de l'Université Jagellonne en 19896.

Si la comtesse Tarnowska commence à tenir son journal à l'occasion d'un voyage, et inscrit donc son texte dans une forme reconnue, elle reprend la plume quelques mois après son retour en Pologne avec une finalité moins immédiatement identifiable. Bien sûr, elle affiche en ouverture un enjeu moral. Elle dit écrire pour «se rappeler [ses fautes] de temps en temps ». Mais l'examen de conscience n'est pas, au long du texte, la première activité de la diariste dont l'intention semble plutôt, d'un côté, de conserver trace des événements marquants de son existence, de celle des membres de sa famille, et dans une certaine mesure de celle de son pays, pour pouvoir s'en souvenir et éventuellement les évoquer devant un cercle de proches, et de l'autre de constituer une histoire de soi qui soit une histoire des impressions que chaque journée lui a laissées, et des sentiments éprouvés. Ces finalités ne partagent pas, toutefois, le texte de façon stricte. La dimension de chronique familiale aristocratique n'est jamais entièrement absente, mais elle se trouve souvent voilée par l'expression intime de l'amour pour son mari ou pour son père, par le regret de ses enfants morts en bas âge ou par les récits de ses terreurs nocturnes. Car le cahier est de façon dominante l'espace où l'émotion retenue peut s'avouer : «J'écris pour remplir, occuper chacun de mes instants... si j'en accorde un seul aux pensées qui me viennent en foule, aussitôt les larmes me suffoquent... et crainte de le donner en spectacle aux indifférents qui m'entourent, je me comprime d'une manière bien pénible », écrit-elle en un moment historique difficile où elle craint pour la sécurité de son mari7.

6. Georges Mycielski, « Une jeune Polonaise en Italie à l'époque du Premier Consul », suivi de «Journal du voyage en Italie de la comtesse Valérie Tarnowska (1803-1804) publié par le comte Georges Mycielski, professeur de l'Histoire de l'art à l'université de Cracovie », Revue de Pologne, 1924, p. 1-53. La publication du journal se continue sur les numéros suivants (1924 : p. 149-178, 484-513; 1925 : p. 61-114 et 281-339). Elle a été achevée en 1989 par Marie Wilczyńska dans la revue de la Bibliothèque Jagellonne : Valérie Tarnowska, « Mes voyages (1804). Deuxième partie éditée et annotée par Marie Wilczynska », Biuletyn biblioteki jagiellonskiej, R. XXXIX, 1989, p. 35-75 (cette seconde partie comporte des erreurs de transcription). Le journal postérieur de la comtesse (1804-1838) n'a jamais été publié. La partie du 12 juin au 25 août 1818 a toutefois fait l'objet d'une édition critique dans le cadre d'un mémoire de maîtrise (Olivier Léric, Waleria Tarnowska, Mon Journal : séjour à SaintPétersbourg (1818). Édition critique, sous la direction de Michel Braud, Université de Bordeaux III, septembre 2005).

7. Vol. III, $\mathrm{f}^{\circ} 1 \mathrm{r}^{\circ}$. 
Waleria Tarnowska, qui avait intitulé son journal de voyage en Italie Mes voyages, intitule d'ailleurs celui-ci Mon Journal, le plaçant ainsi dans un registre d'écriture plus intime. Pour autant, la destination du texte demeure ambiguë, entre espace social et retrait privé : d'un côté la comtesse destine explicitement certaines notes à son mari ou prévoit de lire à ses proches celles qu'elle rédige au cours de ses voyages 8 ; de l'autre, elle envisage de brûler son journal avant sa mort par crainte du ridicule : «Peut-être le brûlerai-je. J'ai été trop sensible, trop exaltée peut-être ma vie durant, et mon fils est trop froidement raisonnable pour que j'aie le courage de m'exposer à lui paraître ridicule ${ }^{9}$. »

Elle le tient néanmoins assez régulièrement pendant trente-quatre ans, c'est-à-dire, avec des lacunes qui peuvent parfois aller jusqu'à six mois ou un an, de 1804 à 1838, sur neuf cahiers de format assez régulier d'environ $19 \mathrm{~cm}$ sur 23, à 1 'exception du dernier de format $21 \times 26 \mathrm{~cm}$. Le nombre de folios n'est pas constant : deux cent trentehuit pour le premier cahier, cent soixante-quinze pour le second, deux cent trente et un pour le troisième, etc. Les cahiers ont été foliotés par volume, mais aussi paginés de façon suivie par la comtesse, de la page 1 à la page 4659 (pour les pages remplies), ce qui dénote de sa part un sentiment de continuité de l'histoire qu'elle rapporte. Le dernier cahier ne comporte que cent douze folios rédigés ; les numéros de folios et pages suivantes ont été portés par avance, ce qui laisse supposer une projection dans l'avenir de l'activité d'écriture qui n'a pas été réalisée. La suspension de l'écriture ne semble pas avoir de cause particulière : aucun événement particulier n'est évoqué, et la comtesse ne mourra que dix ans plus tard.

La reliure en cuir a visiblement été réalisée après la rédaction puisqu'elle cache parfois légèrement la fin des lignes des versos, mais du vivant de la comtesse puisque celle-ci reporte elle-même le titre Mon Journal sur la page de garde de certains volumes. La langue est le français, en dehors de quelques passages en polonais (poème, chanson, mais aussi compte rendu de visite d'un musée). La rédaction est presque entièrement de sa main : seuls quelques passages (en français ou en polonais) sont copiés par l'une de ses filles adoptives - la comtesse précisant à ce propos en une occasion : "C C'est un autre moimême ${ }^{10}$. » La date est donnée dans les deux styles lorsqu'elle voyage en Russie. Comme le journal de voyage en Italie, le manuscrit ne comporte que peu de ratures, sauf en quelques rares endroits où plusieurs mots ou lignes ont été soigneusement biffées à la relecture pour des raisons qui semblent être d'ordre moral, le texte reprenant, par exemple : «Sur sa physionomie riante, je voyais la vertu satisfaite d'elle-même11. » Diverses illustrations sont aussi intégrées au journal : un dessin à l'encre à l'occasion de la visite d'une demeure, et plusieurs gravures de monuments parisiens lors du voyage en France que fait la comtesse dans les années 1824-182612.
Le second journal relève, lui, davantage d'un modèle introspectif car l'analyse des sentiments y est très développée, mais le texte nous est parvenu sous une forme lacunaire. La Bibliothèque Jagellonne ne possède en effet que les tomes cinq, sept et huit du journal d'Eliza Michałowska13, les autres ayant semble-t-il été perdus. Nous n'avons de plus aucune information sur la vie de l'auteur, en dehors de celles que donne son journal.

Le premier des volumes conservés débute en mai 1833 , mais la diariste précise qu'elle se livre à cette activité depuis un an ; le dernier volume s'arrête en octobre 1834. La jeune fille vit à Vienne - la Galicie polonaise est alors, comme on 1'a indiqué, sous domination autrichienne - avec sa mère et sa sœur. Son français est soigné, peu fautif, et l'on sait qu'elle écrit l'allemand (elle rédige pour sa mère une supplique à l'empereur) et étudie l'italien - elle utilise d'ailleurs quelques expressions italiennes dans son discours. Elle attend le mariage, sans en parler jamais - sinon indirectement lorsqu'elle évoque un

8. Voir par exemple les volumes $\mathrm{V}, \mathrm{f}^{\circ} 250 \mathrm{v}^{\circ}$, et VI, fo $82 r^{\circ}$. 9. Vol. V, fo $204 \mathrm{r}^{\circ}$.

10. Vol. IV, $\mathrm{f}^{\circ} 41 \mathrm{r}^{\circ}$.

11. Vol. I, f $\mathrm{f}^{\circ} 126 \mathrm{r}^{\circ}$.

12. Voir sur ce voyage mon article « Le Voyage en France de la comtesse Tarnowska ", dans Alain Guyot et Chantal Massol (dir.), Voyager en France au temps du romantisme. Poétique, esthétique, idéologie, Grenoble, ELLUG, 2003, p. 169-180.

13. Journal d'Eliza Michatowska, t. V, VII et VIII, Bibliothèque de l'Université Jagellonne de Cracovie, cotes 5968/1 à 3. Ce journal n'a jamais été publié. 
rêve de bal ou exprime sa solitude douloureuse, tempérée seulement par l'amour qu'elle porte à sa mère.

Le ton est mélancolique, et Eliza s'interroge sur ce sentiment; elle désirerait paraître enjouée, mais sait que « la mélancolie et les chagrins sont des hôtes fréquents à la nature humaine 14 ». Sa cyclothymie et son penchant pour l'introspection 1 'entraînent parfois vers des questions métaphysiques qui la laissent troublée : «Je tombe dans un chaos d'idées d'où je ne sors que l'esprit troublé. Qu'est-ce que la vie ? Qu'est-ce que la mort ? Où estil ce Dieu éternel15 ? »Sa mélancolie lui fait admirer Chateaubriand et surtout Lamartine, mais elle ne trouve quelque consolation que dans la musique qui est « [son] autre vie, [son] autre âme16 ».

L'origine de son sentiment de lassitude et de tristesse nous est suggérée dans le récit d'un rêve où elle voit son père - mort deux ans auparavant -, et dans les reproches qu'elle se fait de n'avoir pas été assez tendre avec lui. «Le présent n'est rien pour l'homme, écrit-elle, mais quand il est passé ce présent, quand vaguement le souvenir nous le retrace, quand on se demande presque : ce qui n'est plus a-t-il jamais été ? alors ces plaisirs qui ne sont plus, et qui, quand nous pouvions en jouir furent à peine goûtés, c'est alors que ces plaisirs perdus à jamais nous paraissent de célestes joies17 ! » Son regret peut l'amener à rêver fugitivement de rejoindre « dans l'autre séjour » ce père aimé trop tard, mais cette idée est bientôt recouverte par les larmes de l'émotion et du regret, puis par la vie quotidienne, par une visite ou une lecture.

Aucun destinataire n'est indiqué ni même supposé. Le journal d'Eliza Michałowska est un journal strictement intime, comme elle l'indique elle-même à plusieurs reprises. «Personne ne sera initié à ces secrets. Écrivons donc à cœur joie », note-t-elle le 25 mai 1833, pour ajouter le 16 août : «Quand je n'ai ni poésie ni musique pour distraire ma tristesse, écrire est encore mon plus doux plaisir... je n'ai pas d'autre confident de mes peines et de mes plaisirs que ce livre, et je dois encore à cette occupation mes plus doux moments18.»

La jeune femme tient son journal sur des cahiers identiques de 18,5 sur $11,5 \mathrm{~cm}$, et de cent quatre-vingtsept ou cent quatre-vingt-huit folios rédigés, en une écriture large et déliée. Elle a elle-même systématiquement porté sur la page de garde le tome et la date de début de rédaction, ainsi que le titre «Journal » sur un volume, et sur les deux autres « Journal d'Eliza Michałowska », indications qui dénotent à la fois son projet d'écriture de soi et la distance qu'elle prend par l'écriture vis-à-vis de son histoire. Il n'est pas anodin, à ce titre, qu'elle parle à plusieurs reprises, comme on l'a vu dans la citation ci-dessus, de son livre. Elle relit, aussi, soigneusement ses carnets puisqu'elle effectue d'assez nombreuses corrections qui visent en général à atténuer la vivacité de l'expression mélancolique. Si aucun projet littéraire ne s'avoue explicitement - parce qu'il paraîtrait évidemment hors de propos - , toute l'écriture de soi demeure néanmoins dans l'ombre d'une écriture littéraire de l'expérience intérieure.

$*$

Ainsi se révèle le continent des journaux intimes de jeunes filles tenus en français hors de France, au XIXe siècle, à une époque où l'influence culturelle de notre pays était encore très forte, dans cette partie de l'Europe particulièrement. Il reste à poursuivre l'enquête dans $\mathrm{d}$ 'autres bibliothèques polonaises, mais aussi dans les bibliothèques roumaines, et peut-être tchèques, hongroises...

14. T. V (vol. I), f $\mathrm{f}^{\circ} 2 \mathrm{v}^{\circ}$. 15. Id., f $\mathrm{f}^{\circ} 20 \mathrm{r}^{\circ}$.

16. Id., $\mathrm{f}^{\circ} 85 \mathrm{v}^{\circ}$

17. $I d ., \mathrm{f}^{\circ} 91 \mathrm{v}^{\circ}-92 \mathrm{r}^{\circ}$.

18. $I d ., \mathrm{f}^{\circ} 1 \mathrm{r}^{\circ}$ et $187 \mathrm{v}^{\circ}$. 